\title{
Comparison of Standard Conservative Treatment and Early Initiation of Renal Replacement Therapy in Pregnancy-related Acute Kidney Injury: A Single-center Prospective Study
}

\author{
Abhinav Banerjee ${ }^{1}$, Gesu Mehrotra ${ }^{2}$
}

\begin{abstract}
Introduction and objective: Pregnancy-related acute kidney injury (PRAKI) is one of the most important cause of maternal and fetal morbidity and mortality. Some of the reasons behind PRAKI may be due to sepsis, postpartum hemorrhage, preeclampsia, thrombotic microangiopathies (TMA), and acute fatty liver of pregnancy (AFLP). The timing of initiation of renal replacement therapy (RRT) for better patient outcome is still debatable. We conducted this study to establish whether earlier initiation of RRT in PRAKI improves fetomaternal survival compared to a more conservative approach.

Materials and methods: This is a prospective study, where patients were screened at a multispecialty tertiary care hospital in north India for 1 year. The patients were divided into two groups: early RRT and standard AKI treatment. The demographic profile and clinical characteristics of the patients in terms of age, parity access to antenatal care, fetal outcome, urine output, hematological and biochemical profiles, RRT, duration of hospitalization, recovery of renal function, and patients survival were recorded.

Results: This prospective study conducted included 13 patients in the early RRT group and 23 patients in the standard group. Considering the fetal outcome, the number of fetal deaths in early RRT group were $14.29 \%$, whereas for the standard group it was $85.71 \%$. In all, $75 \%$ of early RRT group and $25 \%$ of standard group had normal term delivery. Probability test applied showed they were statistically significant. One maternal mortality was there in the standard group. The indices of maternal outcome in PRAKI patients were found to be statistically insignificant.

Conclusion: The planning of RRT whether early or late does not make a difference in maternal mortality or morbidity. The fetal outcome is significantly better for patients with early RRT than conservative treatment in PRAKI.

Keywords: Fetal outcome, Maternal outcome, Pregnancy-related acute kidney injury, Renal replacement therapy.

Indian Journal of Critical Care Medicine (2020): 10.5005/jp-journals-10071-23537
\end{abstract}

\section{INTRODUCTION}

Pregnancy-related acute kidney injury (PRAKI) has never been standardized and can be defined as a broad range from increase in creatinine or blood urea nitrogen (BUN) to decrease in urine output to need of dialysis. It occurs mainly because of the decrease in renal perfusion or ischemic acute tubular necrosis (ATN) from a variety of physiological disturbances or pathological conditions. ${ }^{1}$

It is one of the most important cause of maternal and fetal morbidity and mortality. An Italian study reported that in the developed world, the incidence of acute kidney injury (AKI) in pregnancy reduced from 1:3,000 to 1:18,000 births from the years 1956-1967 to $1988-1994 .^{2}$ In India, pregnancy-associated AKI requiring dialysis was $15 \%$ in 1982-1991, 10\% from 1992-2002, and has not improved much since then. ${ }^{3}$

However, contrary to the abovementioned data, a recent report from Canada revealed an increasing incidence of PRAKI from 1.66 per 10,000 deliveries between 2003 and 2004, to 2.68 per 10,000 deliveries between 2009 and 2010. 4,5 PRAKI still comprises $25 \%$ of referrals to dialysis centers in developing countries. ${ }^{6}$

The common reasons behind PRAKI may be due to sepsis, hyperemesis gravidarum, postpartum hemorrhage, and renal cortical necrosis (RCN). Some other pregnancy-related conditions, such as preeclampsia, thrombotic microangiopathies (TMA), and acute fatty liver of pregnancy (AFLP), can cause kidney injury. ${ }^{1}$ Septic abortion is the most important cause of PRAKI in developing
${ }^{1}$ Department of Anesthesia and Critical Care, Asian Vivekanand Super Speciality Hospital, Moradabad, Uttar Pradesh, India

${ }^{2}$ Department of Anesthesiology and Critical Care, Siddh Multispecialty Hospital, Moradabad, Uttar Pradesh, India

Corresponding Author: Abhinav Banerjee, Department of Anesthesia and Critical Care, Asian Vivekanand Super Speciality Hospital, Moradabad, Uttar Pradesh, India, Phone: +91 7542033082, e-mail: abhinav.banerjee.in@gmail.com

How to cite this article: Banerjee A, Mehrotra G. Comparison of Standard Conservative Treatment and Early Initiation of Renal Replacement Therapy in Pregnancy-related Acute Kidney Injury: A Single-center Prospective Study. Indian J Crit Care Med 2020;24(8):688-694.

Source of support: Nil

Conflict of interest: None

countries. ${ }^{7}$ Puerperal sepsis and postpartum hemorrhage are the leading causes of acute tubular necrosis (ATN) in the postpartum period. The patients with ATN resulting in renal cortical necrosis are more prone to go for dialysis dependency. ${ }^{8,9}$ Nearly $50-170 \%$ of $\mathrm{AKI}$ are associated with physiological complications of pregnancy of which preeclampsia/eclampsia is the most common cause. ${ }^{10}$

Newer standardized methods of classification of AKI using the RIFLE and AKIN criteria may have led to better diagnosing 
and categorizing PRAKI, with seemingly higher incidence getting tabulated. But reports that describe the burden of PR AKI (PRAKI) is more insufficient because of small sample size, highly selected populations, and use of definitions for AKI that has never been validated in pregnancy. The absolute number of cases of AKI during pregnancy is still significantly lower in developed countries than in developing countries. ${ }^{11}$

The timing of initiation of renal replacement therapy (RRT) for better patient outcome is still debatable with conflicting data from different randomized controlled trials. ${ }^{12-15}$ Although the need to initiate RRT is absolute in patients with life-threatening $\mathrm{AKI}$ and complications that are refractory to medical measures, the advantages of commencing RRT in the absence of such complications and starting them early is still debatable. ${ }^{16,17}$

Earlier initiation of RRT may have benefits such as accelerated achievement of euvolemia, removal of toxic solutes, prevention of metabolic disarrangement, and overt complications attributable to AKI. On the other hand, spontaneous recovery of kidney function may occur in selected patients with severe AKI. So earlier initiation in these patients unnecessarily exposes them to the potential harms of vascular access (for example, hemorrhage, thrombosis, and bacteremia) and the complications of RRT (hypotension, hypersensitivity to the extracorporeal circuit, clearance of trace elements, and antibiotics) along with added resource utilization. ${ }^{16,17}$

However, a recent meta-analysis concluded that early initiation of RRT in AKI does not improve patient survival or intensive care unit (ICU) or hospital length of stay (LOS) when compared to standardized initiation of RRT. ${ }^{18}$

We conducted this current study to establish whether earlier initiation of RRT in the setting of severe AKI in pregnancy improves fetomaternal survival compared to a more conservative approach of waiting and supportive care in which RRT is initiated only when classic indications arises.

\section{Materials and Methods}

This is a prospective study in which patients were screened at a multispecialty tertiary-care hospital in north India. Data of all the cases of PRAKI referred to ICU department in 1 year were considered. Patients were defined for AKI per RIFLE criteria. The demographic profile and clinical characteristics of the patients in terms of age, parity access to antenatal care, fetal outcome, urine output, hematological and biochemical profiles, rRRT, duration of hospitalization, recovery of renal function, and patients survival were recorded.

The patients were divided into two groups:

- Early RRT (RRT done in $<24$ hours)

- Standard AKI treatment with possibility of late RRT if required.

\section{Inclusion Criteria for Patients for RRT}

- Adults $>18$ years age

- Presence of severe AKI defined by the presence of two of the following three criteria:

- A twofold increase in serum creatinine from baseline;

- Urine output $<0.6 \mathrm{~mL} / \mathrm{kg}$ in the preceding 12 hours; or

- Urea more than twofold from the baseline.

\section{Exclusion Criteria}

- Lack of commitment to ongoing life support, including RRT

- Presence of an intoxication requiring extracorporeal removal
- RRT within the previous 2 months

- Clinical suspicion of renal obstruction, rapidly progressive glomerulonephritis, or interstitial nephritis

- Prehospitalization estimated glomerular filtration rate of $30 \mathrm{~mL} /$ minute per $1.73 \mathrm{~m}^{2}$

- The passage of 48 hours since doubling of baseline serum creatinine

- Pregnancy in renal transplant recipients, patients with previous history of renal diseases, diabetes mellitus, or hypertension prior to pregnancy, and those with small or unequal kidneys on ultrasound or any evidences of chronic kidney diseases were excluded from the study.

- Pregnancy with history of septic abortion causing acute kidney injury.

If all of the inclusion criteria were met and no exclusion criteria were present, we considered the participant to be provisionally eligible.

In the standard RRT initiation group, we considered including patients in whom conservative therapy for AKI was considered or RRT was not started within first 24 hours. Patients were also considered in this group who had initial 24-hour window period of dialysis from eligibility elapsed. The reason of starting RRT for them has been noted.

The patients were decided for the treatment/dialysis by the concerned nephrologist after consulting the primary physician (obstetrician). A protocolized approach was considered and data plotted in the master table.

The data were collected by the concerned doctor on duty who is also blinded of the study.

The fetal outcome of pregnancy was noted in terms of preterm, full-term alive, or dead fetus. Maternal outcome is noted in terms of number of maternal death in each group along with length of stay in ICU (LOS), alive with improved kidney function test (KFT), or dialysis dependency after 92 days of follow-up. Preterm delivery was defined by delivery before the 37 completed weeks of pregnancy. The renal recovery was analyzed in terms of achievement of normal renal function at the time of discharge, dialysis dependent, and dialysis independent state of the patients. The study was approved by the ethics committee of the institute.

\section{Statistical Analysis}

All the data were analyzed with statistical tool "MedCalc". Data will be presented as mean with standard deviation or proportions as appropriate. Mean, median, standard deviation, and variance were calculated, and the following statistical significance tests were applied. Student's unpaired $t$ test was used as the statistical tool to test for significance of observed mean differences. Chi-square test was used for statistical significance test and test of significance for difference of proportions. Finally, the calculated value was compared to the tabulated value at particular degree of freedom and finds the level of significance. A " $p$ value" should be considered to be nonsignificant if $>0.05$ and significant if $<0.05$.

\section{Results}

This prospective study conducted included 13 patients in the early RRT group and 23 patients in the standard group. The whole study was done within a time period of 1 year. Percentage of patients in early group were $36.11 \%$ and standard group was $63.89 \%$. The distribution was statistically significant (Table 1). The baseline clinical characteristics were then tabulated in Tables 2 to 4 . It 
consisted demographic (age, weight), clinical (systolic and diastolic blood pressure, and urine output), and lab parameters (platelet, potassium, calcium, hemoglobin, creatinine, and urea levels).

\section{Demographic Parameters (Table 2)}

Considering the other clinical variables, the age and the weight between the two groups were statistically nonsignificant. The mean \pm SD of age and weight were $27 \pm 3.42$ and $53.45 \pm 5.82$ for early RRT group and $28.96 \pm 4.19$ and $52.64 \pm 5.01$ for standard group, respectively.

\section{Clinical Parameters (Table 3)}

The systolic blood pressure (SBP) and diastolic blood pressure (DBP) for early RRT and standard group were 130.48 \pm 16.65 and $85.13 \pm 10.42$ and $107.38 \pm 27.45$ and $63.83 \pm 15.82$, respectively. Initial recordings systolic blood pressure and diastolic blood pressure for both the groups were clinically significant with $p$ value $<0.05$. The urine output was also statistically significant when compared.

\section{Laboratory parameters (Table 4)}

The mean \pm SD of early RRT group of hemoglobin was $8.68 \pm 2.39$ and standard was $9.86 \pm 1.76$. Creatinine was $4.04 \pm 1.93$ for early RRT group and $2.9 \pm 1.19$ for standard group. When proportion test

Table 1: Overall population distribution

\begin{tabular}{lll}
\hline Parameter & Early RRT & Standard group \\
\hline No. of population & 13 & 23 \\
Percentage & 36.11 & 63.89 \\
\hline
\end{tabular}

Table 2: Demographic parameters of patients during pregnancy associated with AKI

\begin{tabular}{|c|c|c|c|c|}
\hline & $\begin{array}{l}\text { Early group } \\
(n=13)\end{array}$ & $\begin{array}{l}\text { Staudard group } \\
(n=23)\end{array}$ & & \\
\hline Variables & Mean $\pm S D$ & Mean $\pm S D$ & $|t|_{\text {cal }}$ & $p$ value \\
\hline Age (years) & $27 \pm 3.42$ & $28.96 \pm 4.19$ & 1.435 & 0.1603 \\
\hline Weight (kg) & $53.45 \pm 5.82$ & $52.64 \pm 5.01$ & 0.440 & 0.6630 \\
\hline ANC & $13(100 \%)$ & $23(100 \%)$ & \multicolumn{2}{|c|}{ Test not applied } \\
\hline
\end{tabular}

applied, they were found to be statistically significant. The other lab parameters such as platelet count, potassium, and calcium were comparable between the two groups.

The etiology of AKI in pregnant patient is categorized in Table 5. Preeclampsia consisted of the maximum number of patients with a total of $36.11 \%$. Patient with sepsis and malaria consisted of a total of nine patients. Some uncommon causes were acute fatty liver of pregnancy and snake bite.

The relative risk of mortality of patients with regard to different symptoms of PRAKI has been documented in Table 6 .

\section{Clinical Outcome (Tables 7 and 8; Figs 1 and 2)}

Considering the fetal outcome, the number of fetal deaths in early RRT group were $14.29 \%$, whereas for the standard group it was $85.71 \%$. In all, $75 \%$ of early RRT group and $25 \%$ of standard group had normal term delivery. Probability test applied showed they were significant statistically.

In regard to maternal outcome, one maternal mortality was observed in the standard group. The length of ICU stay was 9 days in early RRT and 12 days in standard group. The alive and improved KFT and alive and dialysis-dependent percentages were found to be statistically insignificant.

The relative risk of different symptoms in patients with AKI is documented in Table 6 . Almost $63.23 \%$ of the patients in the early RRT group were septic, whereas only $13.04 \%$ were septic in the standard group. The relative risk of sepsis is 5.607, with a probability value $<0.05$. The other symptom that is statistically significant when compared between the two groups was hemorrhagic complication: $60.87 \%$ were in standard group and $15.38 \%$ in the early RRT group. Patients having other symptoms like oliguria, encephalopathy, hepatic dysfunction, low platelet, pulmonary edema, acidosis, high creatinine, urea, and hyperkalemia were statistically insignificant.

\section{Discussion}

The most important concern about pregnancy is the body's dramatic changes caused by gestation and the treatment of two patients simultaneously, with only one directly accessible, the mother. Renal failure in pregnancy has particular challenges because it occurs in a system physiologically altered from baseline

Table 3: Clinical parameters of patients during pregnancy associated with AKI

\begin{tabular}{lccrrr}
\hline & Early group $(n=13)$ & & & \\
\cline { 2 - 2 } Variables & Mean $\pm S D$ & Mean $\pm S D$ & $|t|_{\text {cal }}$ & p value \\
\hline SEP $(\mathrm{mm} \mathrm{Hg})$ & $130.48 \pm 16.65$ & $107.38 \pm 27.45$ & 3.155 & 0.0034 \\
DBP $(\mathrm{mm} \mathrm{Hg})$ & $85.13 \pm 10.42$ & $63.53 \pm 15.82$ & 4.875 & $<0.0001$ \\
Urine output $(\mathrm{mL} /$ day $)$ & $25.75 \pm 50.30$ & $130.39 \pm 189.40$ & 2.870 & 0.007 \\
\hline
\end{tabular}

Table 4: Laboratory parameters of patients during pregnancy associated with AKI

\begin{tabular}{|c|c|c|c|c|}
\hline & Early group $(n=13)$ & Staudard group $(n=23)$ & & \\
\hline Variables & Mean $\pm S D$ & Mean $\pm S D$ & $|t|_{\text {cal }}$ & $p$ value \\
\hline Platelet count (lakh/ $\mu \mathrm{L})$ & $155.56 \pm 110.17$ & $175.78 \pm 125.45$ & 0.434 & 0.6312 \\
\hline Serum potassium (mEq/L) & $4.76 \pm 1.08$ & $4.32 \pm 1.17$ & 1.113 & 0.2734 \\
\hline Serum calcium (mg/dL) & $7.85 \pm 1.01$ & $8.18 \pm 1.04$ & 0.340 & 0.4069 \\
\hline Hemoglobin ( $\mathrm{g} / \mathrm{dL}$ ) & $8.68 \pm 2.39$ & $9.86 \pm 1.76$ & 1.523 & 0.1357 \\
\hline Urea (mmol/L) & $104.5 \pm 11.13$ & $82.5 \pm 9.2$ & 6.35 & $<0.0001$ \\
\hline Serum creatinine $(\mu \mathrm{mol} / \mathrm{L})$ & $4.04 \pm 1.93$ & $2.9 \pm 1.19$ & 2.20 & 0.0347 \\
\hline
\end{tabular}


and also disease processes superimposed that are specific to pregnancy whose pathophysiology are sometimes incompletely understood with the overlapping symptoms. ${ }^{19}$ Pregnancy induces an $80 \%$ increase in renal plasma flow leading to a $50 \%$ rise in glomerular filtration rate (GFR). ${ }^{20}$ Changes occur early and persist until term. ${ }^{21}$ Creatinine clearance is 30\% higher than in non-pregnant women, ${ }^{20}$ resulting in a serum creatinine decrease from nonpregnant values by $20-30 \% .^{21}$

In this study, the nephrologist, the primary physician, and the data collector are blinded of the study. We see $36.11 \%$ of

Table 5: Etiologies of pregnancy associated AKI

\begin{tabular}{lcc}
\hline Causes & No. of patients & Percentage \\
\hline AFLP & 2 & 5.56 \\
Dengue & 1 & 2.73 \\
Eclampsia & 13 & 36.11 \\
HPLLP syndrome & 1 & 2.78 \\
Hyperemesis gravidarum & 2 & 5.56 \\
Malaria & 4 & 11.11 \\
Hemorrhage & 4 & 11.11 \\
Sepsis & 5 & 13.39 \\
Snake bite & 1 & 2.78 \\
Viral sepsis & 3 & 3.33 \\
\hline
\end{tabular}

PRAKI patients had undergone early RRT, whereas $63.89 \%$ patients underwent conservative therapy. Of the 13 patients in early RRT group, 11 (84.62\%) patients were dialyzed because of high creatinine/urea level, whereas the remaining two (15.38\%) patients were dialyzed because of oliguria/anuria/hyperkalemia/ acidosis. Out of the patients who were continued with standard conservative treatment, five (21.73\%) patients went for late RRT. The most important cause of it was pulmonary edema (60\%), while other causes were life-threatening metabolic acidosis (20\%) and hyperkalemia (20\%). Wald et al. in his study demonstrated patients going for RRT among conservative standard group is 63\%. ${ }^{16}$

The difference in the demographics can be attributed to the difference in decision-making by the nephrologist and the primary physician (obstetrician). It has been observed that PRAKI with sepsis was more taken for early RRT (69.23\%), whereas PRAKI with hemorrhage was less frequently (15.38\%) planned. Same is for PRAKI with preeclampsia features, where most of them underwent standard treatment of renal failure (23.07\%). The $p$ value calculated to be statistically significant.

This difference in categorization as observed in our study might due to the thinking that the importance of RRT in a sepsis patient is more than other pregnancy-related pathology. Moreover, hemorrhage or preeclampsia with PRAKI patients are more subjected to the treatment by the obstetrician as primary physician who were more reluctant for dialysis than

Table 6: Risk of symptoms of patients with pregnancy-associated AKI

\begin{tabular}{|c|c|c|c|c|c|c|c|c|}
\hline \multirow[b]{2}{*}{ Symptoms } & \multicolumn{2}{|c|}{ Early group $(n=13)$} & \multicolumn{2}{|c|}{ Staudard group $(n=23)$} & \multirow[b]{2}{*}{$R R$} & \multirow[b]{2}{*}{$|Z|_{\text {cal }}$} & \multirow[b]{2}{*}{$95 \% \mathrm{Cl}$} & \multirow[b]{2}{*}{$p$ value } \\
\hline & No. & (\%) & No. & (\%) & & & & \\
\hline Oliguria $(D=32)$ & 10 & 76.92 & 22 & 95.65 & 0.8042 & 1.377 & $0.5397-1.0967$ & 0.1636 \\
\hline Sepsis ( $D=12)$ & 9 & 69.23 & 3 & 13.04 & 5.307 & 2.932 & $1.7392-16.1979$ & 0.0034 \\
\hline $\operatorname{CXS}(n=6)$ & 3 & 23.08 & 3 & 13.04 & 1.7692 & 0.772 & $0.4156-7.5321$ & 0.4401 \\
\hline Hepatic dysfunction $(n=13)$ & 2 & 15.38 & 11 & 47.83 & 0.3217 & 1.654 & $0.0838-1.234$ & 0.0932 \\
\hline Low platelet $(n=15)$ & 3 & 23.08 & 12 & 52.17 & 0.4423 & 1.499 & $0.1522-1.2854$ & 0.1339 \\
\hline Pulmonary edema $(n=16)$ & 3 & 61.54 & 8 & 34.78 & 1.7692 & 1.585 & $0.8737-3.5828$ & 0.1130 \\
\hline Acidosis $(n=4)$ & 2 & 15.38 & 2 & 8.70 & 1.7692 & 0.608 & $0.2815-11.120$ & 0.5430 \\
\hline High create urea $(n=31)$ & 11 & 84.62 & 21 & 91.30 & 0.9267 & 0.565 & $0.7118-1.2066$ & 0.5720 \\
\hline Hemorrhage $(n=16)$ & 1 & 15.38 & 14 & 60.87 & 0.2527 & 2.048 & $0.0677-0.9427$ & 0.0406 \\
\hline Hyperkalemia $(D=4)$ & 2 & 15.38 & & 8.70 & 1.7692 & 0.608 & $0.2815-11.12$ & 0.5430 \\
\hline Hypertension $(n=16)$ & 4 & 30.77 & 19 & 32.61 & 0.3725 & 2.314 & $0.1613-0.8599$ & 0.0207 \\
\hline
\end{tabular}

Table 7: Fetal outcome

\begin{tabular}{|c|c|c|c|c|c|c|}
\hline \multirow[b]{2}{*}{ Fetal outcome } & \multicolumn{2}{|c|}{ Early group } & \multicolumn{2}{|c|}{ Standard group } & \multirow[b]{2}{*}{$|Z|_{c a l}$} & \multirow[b]{2}{*}{ pvalue } \\
\hline & No. & (\%) & No. & (\%) & & \\
\hline Dead $(n=14)$ & 2 & 14.29 & 12 & 85.71 & 33.925 & $<0.0001$ \\
\hline Preterm $(n=18)$ & 5 & 44.44 & 10 & 55.56 & 0.501 & 0.4790 \\
\hline Normal delivery $(n=4)$ & 3 & 75 & 01 & 25 & 16.056 & $<0.0001$ \\
\hline
\end{tabular}

Table 8: Maternal outcome

\begin{tabular}{|c|c|c|c|c|c|c|}
\hline \multirow[b]{2}{*}{ Mother outcome } & \multicolumn{2}{|c|}{ Early group } & \multicolumn{2}{|c|}{ Standard group } & \multirow[b]{2}{*}{$|Z|_{c a l}$} & \multirow[b]{2}{*}{$p$ value } \\
\hline & No. & (\%) & No. & (\%) & & \\
\hline Dead & 0 & $0 \%$ & 1 & 4.35 & 0.0857 & 0.7697 \\
\hline Length of ICU stay & 9 & 69.23 & 12 & 52.17 & 0.416 & 0.5187 \\
\hline Alive and improved KFT & 11 & 84.62 & 22 & 95.65 & 0.273 & 0.6014 \\
\hline Alive and dialysis dependent & 2 & 15.38 & 1 & 4.35 & 0.273 & 0.6014 \\
\hline
\end{tabular}




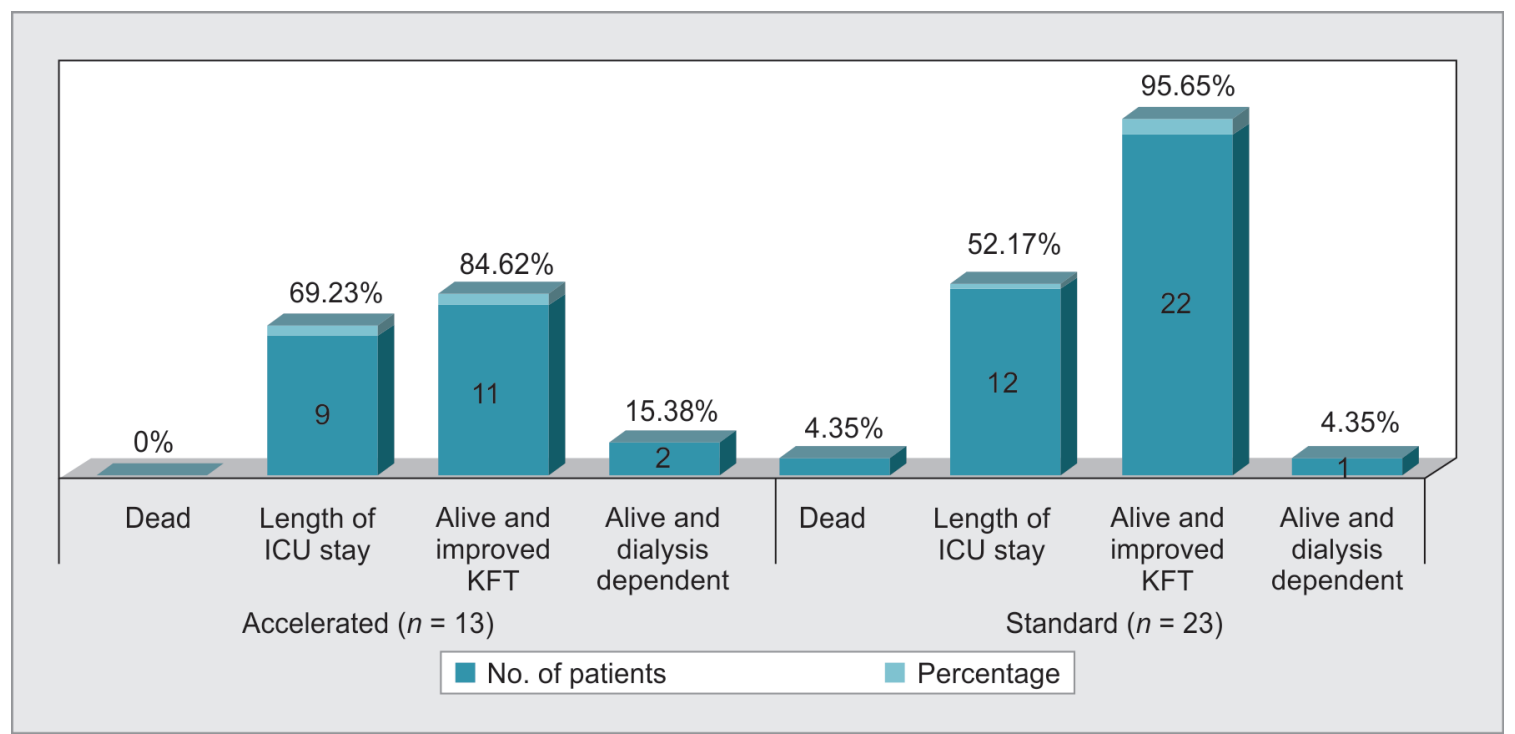

Fig. 1: Maternal outcome in early (accelerated) group and standard group

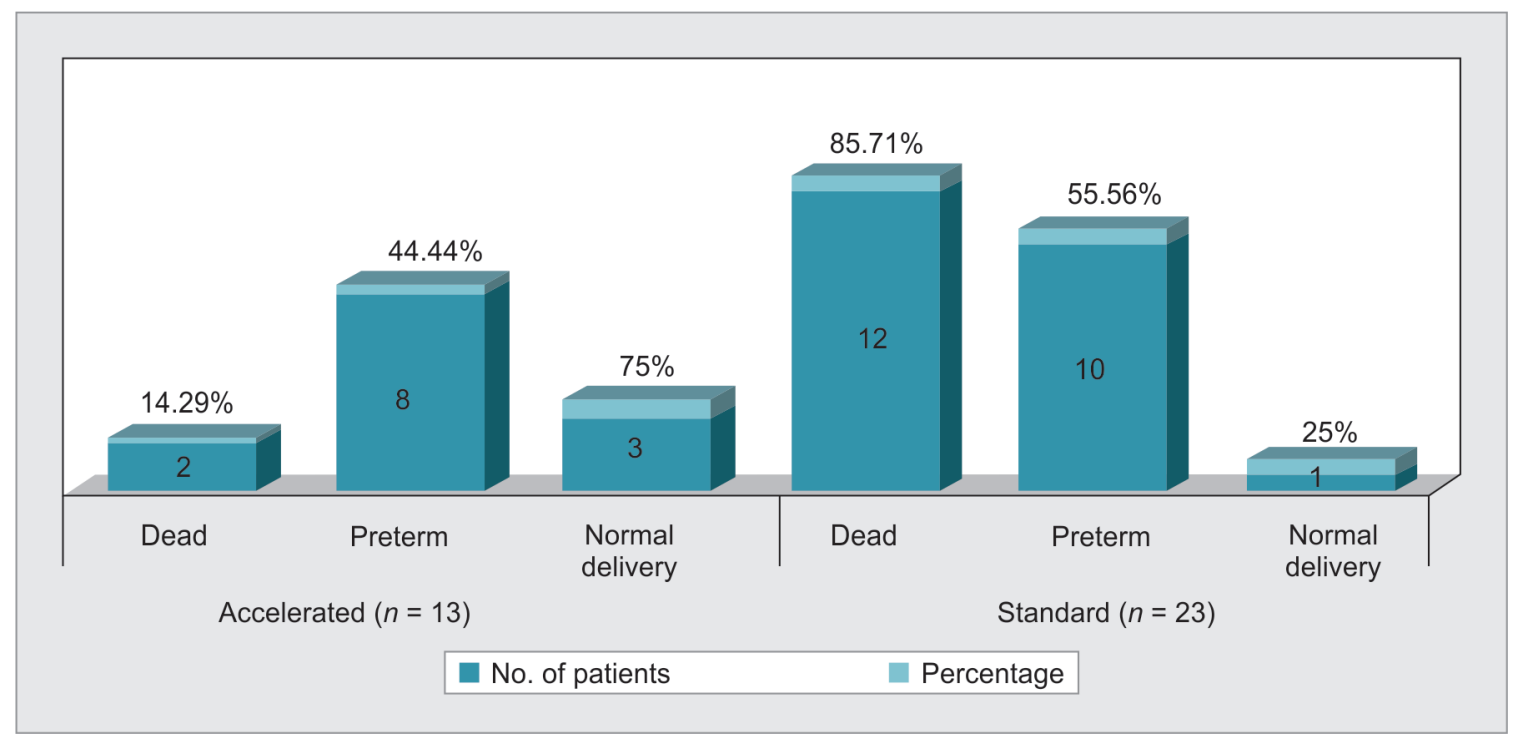

Fig. 2: Fetal outcome in early (accelerated) group and standard group

PRAKI patients with sepsis who were much more often under control of nephrologists. PRAKI patients with other symptoms like oliguria, pulmonary edema, uremic encephalopathy, hepatic dysfunction, and thrombocytopenia were comparable between the early RRT and standard AKI treatment. Hepatic dysfunction and thrombocytopenia were not associated with increased risk of early dialysis in our study, while these factors have shown to be associated with maternal mortality in some other studies. ${ }^{22,23}$

The most common causes of PRAKI in the early trimester is prerenal because of hyperemesis gravidarum and septic abortion causing sepsis. ${ }^{1}$ During the late trimester, most causes of PRAKI involve hypertensive diseases in pregnancy, (thrombotic microangiopathies) TMA, (acute fatty liver of pregnancy) AFLP, and postpartum hemorrhage. ${ }^{1}$

Pregnancy-related hypertensive disorder remains the leading causes of PRAKI in developed countries, and the decline in PRAKI recently is mainly because of significant decrease in sepsis. ${ }^{2,24,25}$
A study from Casablanca reported that preeclampsia and eclampsia accounts for $74.5 \%$ cases, sepsis in $11 \%$, and hemorrhage for $7.2 \%$ PRAKI cases in developed countries. ${ }^{24}$

In our study, hypertensive disorder consists of $36.11 \%$, $22.22 \%$ having sepsis that includes both viral and bacterial. A good number of PRAKI patients were also diagnosed with malaria and other tropical fever. They can also be collectively considered under septic patients if not differentiated otherwise. Maternal hemorrhage consist $11.11 \%$. All the septic patients that we encountered in our study were systemic infection rather than abortal septicemia as that was an exclusion criteria. AFLP in two (5.56\%) patients. One patient had a snake bite with acute renal failure and also had intrauterine death. These differences in different studies mainly depend on the demographics of the place where cases were studied.

In PRAKI patients, fetal outcome is consistently poor, with high prematurity, polyhydramnios, and fetal growth retardation. ${ }^{26}$ Less 
than $50 \%$ of pregnancies result in a surviving infant, ${ }^{27}$ suggesting a deleterious fetal effect of an azotemic intrauterine environment.

A fetal death of only $5.5 \%$ has been reported in case of PRAKI in a study from a developed country. The fetal mortality of $38.88 \%$ was observed in our study, much higher than reported in pregnant women admitted in ICU with renal failure. ${ }^{28}$ Considering the clinical outcome, we find in our study that the number of fetal deaths is significantly more in the standard acute renal failure treatment than the early RRT group. The number of premature, preterm birth is nearly equal, with the patients who were given early RRT support had more number of normal deliveries. The result is also statistically significant.

The literature on techniques to improve fetal outcome is less, but existing evidence suggests that successful pregnancies and improved fetal outcomes require intensified dialysis regimens. ${ }^{26,27,29}$ Majority of the studies are confined to chronic renal failure. A U.S. survey of 344 pregnancies showed a non-significant trend toward improved gestational age and infant survival with dialysis $>20$ hour/week. ${ }^{30}$ Bagon and colleagues ${ }^{26}$ found similar findings in their national survey of dialysis centers in Belgium in 1998. In an attempt to minimize the effects of uremia on fetus, they adjusted dialysis regimens with the goal to attain maximum predialysis urea levels of $100 \mathrm{mg} / \mathrm{dL}$. A positive correlation was noted between birth weight and excess dialysis hours (the number of hours of dialysis delivered minus the hours that would have been given if the patient were not pregnant). ${ }^{26}$ These two publications have influenced the prescription of dialysis for PRAKI patients internationally, with the recommendation that an early and increased dialysis dose should be standard of care for pregnant women. There have been case reports since then of excellent perinatal outcome with intensive dialysis. ${ }^{31}$ The intensive daily dialysis achieves a physiological state close to the normal pregnant state as possible, thereby providing a theoretical reason to justify its use.

A study from a developed country reported maternal mortality of $9.1 \%$ in case of PRAKI, which is almost half of that reported from India. ${ }^{28}$ In our study while comparing the maternal outcome in regard to the maternal mortality, length of stay in ICU, dialysis dependency, and recovery of renal function after 92 days all seem to be comparable in-between the two groups. There is no benefit to start and initiate RRT early, which can even be termed as an unnecessary RRT if we consider the maternal health outcome solely. A recent met analysis also concluded similar result but that is for nonpregnant kidney failure patients. ${ }^{17}$

\section{Conclusion}

- The outcome of pregnant patients with conservative treatment for PRAKI is comparable to that of patients with early RRT. (alive, dialysis dependent and independent, LOS, death). There exists no difference. So the planning of RRT whether early or late does not make a difference in maternal mortality or morbidity.

- The fetal outcome is significantly better for patients with early RRT than conservative treatment in PRAKI patients.

- We have taken only a small sample of 36 patients. More studies are required to proof the facts as concluded in our study

\section{References}

1. Siribamrungwong $M$, Chinudomwong. P. Relation between acute kidney injury and pregnancy-related factors. J Acute Dis 2016;5(1):2228. DOI: 10.1016/j.joad.2015.08.002.
2. Stratta P, Besso L, Canavese C, Grill A, Todros T, Benedetto C, et al. Is pregnancy-related acute renal failure a disappearing clinical entity? Ren Fail 1996;18(4):575-584. DOI: 10.3109/08860229609047680.

3. Prakash J, Kumar H, Sinha DK, Kedalaya PG, Pandey LK, Srivastava $\mathrm{PK}$, et al. Acute renal failure in pregnancy in a developing country: twenty years of experience. Ren Fail 2006;28(4):309-313. DOI: 10.1080/08860220600583658.

4. Pertuiset N, Grünfeld JP. Acute renal failure in pregnancy. Baillieres Clin Obstet Gynaecol 1994;8(2):333-351. DOI: 10.1016/s09503552(05)80324-4.

5. Chugh KS. Etiopathogenesis of acute renal failure in the tropics. Ann Natl Acad Med Sci (India) 1987;3:88-99.

6. Kilari SK, Chinta RK, Vishnubhotla SK. Pregnancy related acute renal failure. J Obstet Gynecol India 2006;56:308-310.

7. Prakash J, Tripathi K, Malhotra V, Kumar O, Srivastava PK. Acute renal failure in eastern India. Nephrol Dial Transplant 1995;10(11):20092012.

8. Maikranz P, Katz Al. Acute renal failure in pregnancy. Obstet Gynecol Clin North Am 1991;18(2):333-343.

9. Prakash J, Tripathi K, Pandey LK, Gadela SR. Usha renal cortical necrosis in pregnancy-related acute renal failure. J Indian Med Assoc 1996;94(6):227-229.

10. Prakash J, Vohra R, Wani IA, Murthy AS, Srivastva PK, Tripathi K, et al. Decreasing incidence of renal cortical necrosis in patients with acute renal failure in developing countries: a single-centre experience of 22 years from Eastern India. Nephrol Dial Transplant 2007;22(4):12131217. DOI: $10.1093 / \mathrm{ndt} / \mathrm{gfl} 761$.

11. Mehrabadi A, Liu S, Bartholomew S, Hutcheon JA, Magee LA, Kramer $M S$, et al. Hypertensive disorders of pregnancy and the recent increase in obstetric acute renal failure in Canada: population based retrospective cohort study. BMJ 2014;349(jul30 2):g4731. DOI: 10.1136/ bmj.g4731.

12. Jamale TE, Hase NK, Kulkarni M, Pradeep KJ, Keskar V, Jawale S, et al. Earlier-start versus usual-start dialysis in patients with communityacquired acute kidney injury: a randomized controlled trial. Am J Kidney Dis 2013;62(6):1116-1121. DOI: 10.1053/j.ajkd.2013.06.012.

13. Bouman CSC, Oudemans-Van Straaten HM, Tijssen JGP, Zandstra DF, Kesecioglu J. Effects of early high-volume continuous venovenous hemofiltration on survival and recovery of renal function in intensive care patients with acute renal failure: a prospective, randomized trial. Crit Care Med 2002;30(10):2205-2211. DOI: 10.1097/00003246200210000-00005.

14. Gaudry S, Hajage D, Schortgen F, Martin-Lefevre L, Pons B, Boulet $\mathrm{E}$, et al. Initiation strategies for renal-replacement therapy in the intensive care unit. N Engl J Med 2016;375(2):122-133. DOI: 10.1056/ NEJMoa1603017.

15. Zarbock A, Gerß J, Van Aken H, Boanta A, Kellum JA, Meersch M. Early versus late initiation of renal replacement therapy in critically ill patients with acute kidney injury (the ELAIN-trial): Study protocol for a randomized controlled trial. Trials 2016;17(1):148. DOI: 10.1186/ s13063-016-1249-9.

16. Wald R, Adhikari NK, Smith OM, Weir MA, Pope K, Cohen A, et al. Comparison of standard and accelerated initiation of renal replacement therapy in acute kidney injury. Kidney Int 2015;88(4):897-904. DOI: 10.1038/ki.2015.184.

17. Bhatt GC, Das RR. Early versus late initiation of renal replacement therapy in patients with acute kidney injury-a systematic review \& meta-analysis of randomized controlled trials. BMC Nephrol 2017;18(1):78. DOI: 10.1186/s12882-017-0486-9.

18. Wierstra BT, Kadri S, Alomar S, Burbano X, Barrisford GW, Kao RLC. The impact of "early" versus "late" initiation of renal replacement therapy in critical care patients with acute kidney injury: a systematic review and evidence synthesis. Crit Care 2016;20(1):122. DOI: 10.1186/ s13054-016-1291-8.

19. Gammill HS, Jeyabalan A. Acute renal failure in pregnancy. Crit Care Med 2005;33((Suppl.):S372-S384. DOI: 10.1097/01. ccm.0000183155.46886.c6. 
20. Davison JM, Dunlop W. Renal haemodynamics and tubular function in normal human pregnancy. Kidney Int 1980;18(2):152. DOI: 10.1038/ ki.1980.124.

21. Sturgiss SV, Dunlop W, Davison JM. Renal hemodynamics and tubular function in human pregnancy. Baillieres Clin Obstet Gynaecol 1994;8(2):209. DOI: 10.1016/s0950-3552(05)80319-0.

22. Goplani K, Shah P, Gera D, Gumber M, Dabhi M, Feroz A, et al. Pregnancy-related acute renal failure: a single-center experience. Indian J Nephrol 2008;18(1):17-21. DOI: 10.4103/0971-4065. 41283.

23. Krishna A, Singh R, Prasad N, Gupta A, Bhadauria D, Kaul A, et al. Maternal, fetal and renal outcomes of pregnancy-associated acute kidney injury requiring dialysis. 2015;25(Issue 2):77-81. DOI: 10.4103/0971-4065.136890.

24. Hachim K, Badahi K, Benghanem M, Fatihi EM, Zahiri K, Ramdani B, et al. Obstetrical acute renal failure. Experience of the nephrology department, central university hospital Ibn Rochd, Casablanca. Nephrologie 2001;22(1):29-31.
25. Chugh KS, Krishna CR, Sharma BK. ARF of obstetric origin. J Obstet Gynecol 1976;108:253-261.

26. Bagon JA, Vernaeve H, De Muylder X, Lafontaine JJ, Martens J, Van Roost G. Pregnancy and dialysis. Am J Kidney Dis 1998;31(5):756-765. DOI: 10.1016/s0272-6386(98)70060-5.

27. Hou S. Pregnancy in dialysis patients: where do we go from here? Semin Dial 2003;16(5):376-378. DOI: 10.1046/j.1525-139x.2003.16083.x.

28. Naylor DF, Olson MM. Critical care obstetrics and gynecology. Crit Care Clin 2003;19(1):127-149. DOI: 10.1016/s0749-0704(02)00059-3.

29. Gangji AS, Windrim R, Gandhi S, Silverman JA, Chan CM. Successful pregnancy with nocturnal hemodialysis. Am J Kidney Dis 2004;44(5):912-916. DOI: 10.1016/S0272-6386(04)01090-X.

30. Okundaye I, Abrinko P, Hou S. Registry of pregnancy in dialysis patients. Am J Kidney Dis 1998;31(5):766-773. DOI: 10.1016/s02726386(98)70044-7.

31. Prasad S, Parkhurst D, Morton MR, Henning P, Lawton J, Bannister $\mathrm{K}$. Increased delivery of haemodialysis assists successful pregnancy outcome in end-stage renal failure. Nephrology 2003;8(6):311-314. DOI: 10.1111/j.1440-1797.2003.00208.x. 